\title{
Evaluation of the National Electronic Disease Surveillance System Amid the COVID-19 Pandemic in Elsahel District, Cairo Governorate, Egypt, 2020
}

\author{
Neven Girgis; Wessam Elnahry; Salma Afifi; Sahar El Shourbagy; Hanaa Abu Elsood; Alaa Eid
}

Ministry of Health and Population Egypt, Cairo, Egypt

\section{Corresponding Author:}

Neven Girgis

Ministry of Health and Population Egypt

156 Shoubra Street

Cairo, 11672

Egypt

Phone: 2001228604323

Email: nevengirgis72@gmail.com

\section{Abstract}

Background: The Egypt National Disease Surveillance is a routine system established in 2002. The system electronically reports on 41 infectious diseases including COVID-19. Reporting sites include all Egyptian governorates, districts, governmental infectious disease hospitals, and primary health units. Surveillance is essential during the pandemic to detect cases early, describe the epidemiology of health problems, guide priority setting, and plan and evaluate public health policy and strategies.

Objective: This study aims to evaluate the surveillance system during the pandemic to assess its effectiveness in achieving its objectives and to find and fill the gaps.

Methods: The evaluation was performed using the Centers for Disease Control and Prevention guidelines. A structured questionnaire was used to evaluate the qualitative attributes including simplicity, flexibility, and acceptability through interviewing surveillance teams at the central level, health directorate, and Sahel district. Quantitative attributes, including completeness, timeliness, and predictive positive value, were performed using COVID-19 surveillance data of Sahel district in March-December 2020. Data were assessed for completeness and accuracy. The usefulness of surveillance was assessed in terms of achieving its objectives and use of data.

Results: Of 33 respondents, $90 \%$ thought that the system was simple, and $77 \%$ thought it was acceptable; work overload reduced the acceptability rate. The system is funded by the Ministry of Health and Population and was operational $53 \%$ of the time due to connectivity problems. The system was flexible when adapting to include COVID-19 in a short time with minimal cost. It is quite representative, as it covers $60 \%$ of the population. Completeness was $82 \%$, positive value predictive was $58 \%$, and data validity was $86 \%$. The median duration between patient admissions and reporting was 2.7 days.

Conclusions: The evaluation of the Egypt COVID-19 surveillance system indicated that the system partly achieved its objectives in the area of simplicity and flexibility with adequate data quality. There is a need to improve acceptability and timeliness through increasing manpower and to enhance stability through effective connectivity. Expansion of the system to cover all of the Egyptian population is recommended to improve representativeness.

(iproc 2022;8(1):e36514) doi: $10.2196 / 36514$

\section{KEYWORDS}

NEDSS; evaluation; Egypt; surveillance; COVID-19; National Disease Surveillance 
Edited by Y Khader; this is a non-peer-reviewed article. Submitted 16.01.22; accepted 18.01.22; published 04.02.22.

Please cite as:

Girgis N, Elnahry W, Afifi S, El Shourbagy S, Abu Elsood H, Eid A

Evaluation of the National Electronic Disease Surveillance System Amid the COVID-19 Pandemic in Elsahel District, Cairo Governorate, Egypt, 2020

iproc 2022;8(1):e36514

URL: https://www.iproc.org/2022/1/e36514

doi: $10.2196 / 36514$

PMID:

CNeven Girgis, Wessam Elnahry, Salma Afifi, Sahar El Shourbagy, Hanaa Abu Elsood, Alaa Eid. Originally published in Iproceedings (https://www.iproc.org), 04.02.2022. This is an open-access article distributed under the terms of the Creative Commons Attribution License (https://creativecommons.org/licenses/by/4.0/), which permits unrestricted use, distribution, and reproduction in any medium, provided the original work, first published in Iproceedings, is properly cited. The complete bibliographic information, a link to the original publication on https://www.iproc.org/, as well as this copyright and license information must be included. 